\title{
Asymptotics and Numerics of Polynomials Used in Tricomi and Buchholz Expansions of Kummer Functions
}

\author{
José Luis López \\ Departamento de Ingeniería Matemática e Informática, \\ Universidad Pública de Navarra, 31006-Pamplona, Spain \\ Nico M. Temme \\ Centrum Wiskunde \& Informatica, \\ Science Park 123, 1098 XG Amsterdam, The Netherlands. \\ e-mail: jl.lopez@unavarra.es, nicot@cwi.nl
}

July 13, 2010

\begin{abstract}
Expansions in terms of Bessel functions are considered of the Kummer function ${ }_{1} F_{1}(a ; c, z)$ (or confluent hypergeometric function) as given by Tricomi and Buchholz. The coefficients of these expansions are polynomials in the parameters of the Kummer function and the asymptotic behavior of these polynomials for large degree is given. Tables are given to show the rate of approximation of the asymptotic estimates. The numerical performance of the expansions is discussed together with the numerical stability of recurrence relations to compute the polynomials. The asymptotic character of the expansions is explained for large values of the parameter $a$ of the Kummer function.
\end{abstract}

2000 Mathematics Subject Classification: 33C10, 33C15, 41A30, 41A60, 65D20.

Keywords \& Phrases: Kummer functions, confluent hypergeometric functions, Bessel functions, asymptotic expansions, computation of special functions.

\section{Introduction}

The expansions are in terms of the function $E_{\nu}(z)$ which is related to the ordinary Bessel function of the first kind. In Tricomi's notation [15, p. 34] 
we have

$$
E_{\nu}(z)=z^{-\frac{1}{2} \nu} J_{\nu}(2 \sqrt{z})=\sum_{k=0}^{\infty}(-1)^{k} \frac{z^{k}}{k ! \Gamma(\nu+k+1)} .
$$

It is an entire function of $z$. In terms of the modified Bessel function we can write

$$
E_{\nu}(-z)=z^{-\frac{1}{2} \nu} I_{\nu}(2 \sqrt{z})=\sum_{k=0}^{\infty} \frac{z^{k}}{k ! \Gamma(\nu+k+1)} .
$$

We consider expansions of the confluent hypergeometric function (also called Kummer function $)^{1}$

$$
{ }_{1} F_{1}\left(\begin{array}{l}
a \\
c
\end{array} ; z\right)=\sum_{k=0}^{\infty} \frac{(a)_{k}}{(c)_{k}} \frac{z^{k}}{k !} .
$$

Here, $(a)_{k}$ is the Pochhammer symbol defined by

$$
(a)_{0}=1, \quad(a)_{k}=\frac{\Gamma(a+k)}{\Gamma(a)}, \quad k \geq 1 .
$$

Tricomi $[15, \S 1.8]$ has given two expansions in terms of the function $E_{\nu}(z)$, and Buchholz $[4, \S 7.4]$ has given a third example.

\section{Tricomi 1}

$$
{ }_{1} F_{1}\left(\begin{array}{l}
a \\
c
\end{array} ; z\right)=\Gamma(c) e^{\frac{1}{2} z} \sum_{n=0}^{\infty} 2^{-n} z^{n} A_{n}(a, c) E_{c-1+n}(\kappa z),
$$

where

$$
\kappa=\frac{1}{2} c-a,
$$

and the first few coefficients $A_{n}$ are given by

$$
A_{0}(a, c)=1, A_{1}(a, c)=0, A_{2}(a, c)=\frac{1}{2} c, A_{3}(a, c)=-\frac{1}{3}(c-2 a) .
$$

They satisfy the recurrence relation

$$
(n+1) A_{n+1}=(n+c-1) A_{n-1}-2 \kappa A_{n-2}, \quad n \geq 2,
$$

\footnotetext{
${ }^{1}$ This notation for the Kummer function is equivalent with the notation ${ }_{1} F_{1}(a ; c ; z)$ (also used here when appearing in the text) of [13] and $M(a, c, z)$ of [3].
} 
and have the generating function

$$
e^{2 \kappa w}(1-w)^{\kappa-\frac{1}{2} c}(1+w)^{-\kappa-\frac{1}{2} c}=\sum_{n=0}^{\infty} A_{n}(a, c) w^{n}, \quad|w|<1 .
$$

When $c=2 a$, that is, $\kappa=0$, the right-hand side of (1.5) becomes the power series expansion of the modified Bessel function. This follows from $E_{2 a-1+n}(0)=1 / \Gamma(n+2 a)$ and

$$
A_{2 n}(a, 2 a)=\frac{(a)_{n}}{n !}, \quad A_{2 n+1}(a, 2 a)=0, \quad n=0,1,2, \ldots
$$

This gives

$$
{ }_{1} F_{1}\left(\begin{array}{c}
a \\
2 a
\end{array} ; z\right)=\Gamma\left(a+\frac{1}{2}\right) e^{\frac{1}{2} z}\left(\frac{1}{4} z\right)^{\frac{1}{2}-a} I_{a-\frac{1}{2}}\left(\frac{1}{2} z\right),
$$

which is a known identity for the Kummer function.

\section{Tricomi 2}

$$
{ }_{1} F_{1}\left(\begin{array}{l}
a \\
c
\end{array} ; z\right)=\Gamma(c) e^{h z} \sum_{n=0}^{\infty} B_{n}(a, c ; h) z^{n} E_{c-1+n}(-a z) .
$$

The first few coefficients $B_{n}$ are given by

$$
\begin{aligned}
& B_{0}(a, c ; h)=1, \quad B_{1}(a, c ; h)=-c h, \\
& B_{2}(a, c ; h)=-\frac{1}{2}(2 h-1) a+\frac{1}{2} c(c+1) h^{2} .
\end{aligned}
$$

They satisfy the recurrence relation

$$
\begin{aligned}
& (n+1) B_{n+1}=((1-2 h) n-h c) B_{n}+ \\
& \quad((1-2 h) a-h(h-1)(c+n-1)) B_{n-1}-h(h-1) a B_{n-2},
\end{aligned}
$$

$n \geq 2$, and have the generating function

$$
e^{-a w}(1+(h-1) w)^{-a}(1+h w)^{a-c}=\sum_{n=0}^{\infty} B_{n}(a, c ; h) w^{n} .
$$

This series converges for $|w|<\min (1 /|h|, 1 /|1-h|)$. 


\section{Buchholz}

$$
{ }_{1} F_{1}\left(\begin{array}{l}
a \\
c
\end{array} ; z\right)=\Gamma(c) e^{\frac{1}{2} z} \sum_{n=0}^{\infty} 2^{-n} z^{n} P_{n}(c ; z) E_{c-1+n}(\kappa z),
$$

where $\kappa$ is given in (1.6). The first few coefficients $P_{n}$ are given by

$$
\begin{aligned}
& P_{0}(c ; z)=1, \quad P_{1}(c ; z)=\frac{1}{6} z, \quad P_{2}(c ; z)=\frac{1}{72}\left(z^{2}+12 c-24\right), \\
& P_{3}(c ; z)=\frac{1}{6480} z\left(5 z^{2}+180 c-432\right) .
\end{aligned}
$$

They satisfy the recurrence relation [1] (we write $P_{n}(z)=P_{n}(c ; z)$ )

$$
P_{n}(z)=z^{-\frac{1}{2} n} \int_{0}^{z}\left(\frac{1}{4} u P_{n-1}(u)+(c-2) P_{n-1}^{\prime}(u)-u P_{n-1}^{\prime \prime}(u)\right) u^{\frac{1}{2} n-1} d u,
$$

where $n \geq 1$, and have the generating function

$$
e^{-\frac{1}{2} z(\operatorname{coth} w-1 / w)}\left(\frac{\sinh w}{w}\right)^{c-2}=\sum_{n=0}^{\infty}(-1)^{n} P_{n}(c ; z) w^{n}, \quad|w|<\pi .
$$

From this expansion it follows that

$$
P_{n}(c ;-z)=(-1)^{n} P_{n}(c ; z) .
$$

In $\S 2$ we discuss the numerical aspects of these coefficients, in particular the numerical stability of the recurrence relations for computing them. We also consider the asymptotic nature for large values of $a$ of the expansions in (1.5) and (1.16), and our main conclusion is that Buchholz' expansion gives a good numerical performance. In $\S \S 3,4$ we give the large $n$ asymptotic behaviour of the coefficients $A_{n}$ and $P_{n}$, together with tables showing the rates of approximation.

Remark 1 The expansions in (1.5), (1.12), and (1.16) are in fact for the ratio ${ }_{1} F_{1}(a ; c ; z) / \Gamma(c)$, which is well-defined for $c=-m, m=0,1,2, \ldots$ We have

$$
\lim _{c \rightarrow-m} \frac{1}{\Gamma(c)}{ }_{1} F_{1}\left(\begin{array}{l}
a \\
c
\end{array} ; z\right)=\frac{(a)_{m+1} z^{m+1}}{(m+1) !}{ }_{1} F_{1}\left(\begin{array}{c}
a+m+1 \\
m+2
\end{array} ; z\right) .
$$

Remark 2 In the expansions in (1.5) and (1.16) the same Bessel functions are used, and we might wonder if

$$
P_{n}(c ; z) \stackrel{?}{=} A_{n}(a, c),
$$


but comparing the first few values of $P_{n}$ and $A_{n}$ and the completely different nature of the generating functions for these coefficients, we conclude that this is not true.

Remark 3 The expansions in (1.5) and (1.12) are mentioned in [3, p. 506] and $[13, \S 3.8]$, in the latter also with proofs. Analytical aspects of the expansions in (1.5) and (1.12) are discussed in [5], without details of numerical experiments. Tricomi's expansion (1.5) is used in [11] for the computation of parabolic cylinder functions. In [1] and [2] Buchholz' expansion is modified to give an asymptotic expansion for the $U$-Kummer function in terms of $K$-Bessel functions. In [1] several properties of the polynomials $P_{n}(c ; z)$ are discussed, including a useful recurrence relation.

Remark 4 In [3, p. 506] another expansion in terms of Bessel functions is given:

$$
{ }_{1} F_{1}\left(\begin{array}{l}
a \\
c
\end{array} ; z\right)=\sum_{n=0}^{\infty} C_{n}(a, c) I_{n}(z)
$$

where the coefficients satisfy the recursion

$$
C_{n+1}(a, c)=2 a C_{n}(a+1, c+1) / c-C_{n-1}(a, c),
$$

with initial values $C_{0}(a, c)=1, C_{1}(a, c)=2 a / c$. Observe that this recursion is not a true recursion with fixed parameters $a$ and $c$, which is inconvenient for numerical computations, and that an explicit expression for investigating the asymptotic behavior of $C_{n}(a, c)$ seems not to be available. Also, the Kummer function and the Bessel functions in (1.23) have the same argument, whereas the Bessel functions in the expansions in (1.5) and (1.16) have arguments depending on the parameters, which is interesting in connection with the asymptotic nature of these expansions. For expansions in terms of Bessel functions with argument $\frac{1}{2} z$ we refer to $[13, \S 2.7]$, where also proofs are given.

Remark 5 We don't give details about the coefficients $B_{n}$ in (1.12), because we can use the same methods as to be given for $A_{n}$. In addition, the extra parameter $h$ makes the analysis more complicated, and in practice one may choose $h=\frac{1}{2}$ as an obvious value. But then the expansion in (1.5) is of more interest because the argument of the function $E_{\nu}$ in that expansion corresponds better with certain asymptotic expansions of the Kummer function. 


\section{Numerical and asymptotical aspects of the ex- pansions}

We discuss aspects of the rate of convergence of the expansions in (1.5) and (1.16) and the stability of algorithms for computing the coefficients $A_{n}(a, c)$ and $P_{n}(c ; z)$ by recursion. We also consider the use of the expansions as asymptotic expansions for large values of the parameters.

\section{$2.1 \quad$ Numerical aspects}

First we observe that the sequence of Bessel functions occurring in the expansions in (1.5) and (1.16) can be computed by a backward recurrence scheme (the Miller algorithm). For details we refer to $[6, \S 4.6]$.

For large values of $\nu$ (and all $z$ such that $z / \nu=o(1)$ ) the function $E_{\nu}(z)$ defined in (1.1) behaves as $1 / \Gamma(\nu+1)$, and it follows from the asymptotic expansions given in $\S 3$ that the terms in Tricomi's expansion (1.5) behave like

$$
2^{-n} z^{n} A_{n}(a, c) E_{c-1+n}(\kappa z)=\mathcal{O}\left(\frac{(z / 2)^{n} n^{\alpha}}{n !}\right), \quad n \rightarrow \infty,
$$

for some value $\alpha$ that depends on $a$ and $c$. For Buchholz' expansion in (1.16) we have, using the asymptotic expansions given in $\S 4$,

$$
2^{-n} z^{n} P_{n}(c ; z) E_{c-1+n}(\kappa z)=\mathcal{O}\left(\frac{(z / 2)^{n} n^{\beta}}{\pi^{n} n !}\right), \quad n \rightarrow \infty,
$$

for some value $\beta$. Without doubt, because of the factor $\pi^{n}$ in (2.2), Buchholz' expansion has a better rate of convergence than Tricomi's expansion. The significant difference with respect to large- $n$ behavior of $A_{n}(a, c)$ and $P_{n}(c ; z)$ also follows from Table 3 and Table 4 given in $\S \S 3,4$.

On the other hand, for numerical computations the quantities $A_{n}(a, c)$ are easy to obtain by using the recurrence relation given in (1.8). For $P_{n}(c ; z)$ the recurrence relation given in (1.18) is available, but this one is more difficult to use in a numerical algorithm. Using Maple or Mathematica it is easy to generate successive $P_{n}(c ; z)$ from (1.18), and it is possible to generate a large number of these $P_{n}(c ; z)$ for storage.

However, in a numerical algorithm the polynomials $P_{n}(c ; z)$ can be computed by using recurrence relations for its coefficients. Let us write

$$
P_{n}(c ; z)=\sum_{m=0}^{n} p_{n, m} z^{m} .
$$


Table 1: Relative errors $\delta$ in the computation of exact values $P_{n}(c ; z)$ (by using the generating function (1.19)) and finite precision values (by using system (2.4)) for several values of $z, c$ and $n$.

\begin{tabular}{rrccrcc}
\hline \multicolumn{4}{c}{$c=17 / 4$} & \multicolumn{4}{c}{$c=-17 / 4$} \\
\hline$n=50$ & \multicolumn{1}{c}{$P_{n}(c ; z)$} & $\delta$ & \multicolumn{1}{c}{$z$} & $P_{n}(c ; z)$ & $\delta$ \\
& 1 & $-0.7605 \mathrm{e}-28$ & $0.10 \mathrm{e}-18$ & 1 & $0.7873 \mathrm{e}-22$ & $0.18 \mathrm{e}-18$ \\
& 5 & $-0.2581 \mathrm{e}-24$ & $0.19 \mathrm{e}-18$ & 5 & $0.2010 \mathrm{e}-20$ & $0.20 \mathrm{e}-18$ \\
& 10 & $0.6326 \mathrm{e}-23$ & $0.85 \mathrm{e}-17$ & 10 & $-0.6819 \mathrm{e}-19$ & $0.21 \mathrm{e}-16$ \\
& 25 & $0.7183 \mathrm{e}-19$ & $0.32 \mathrm{e}-14$ & 25 & $0.8530 \mathrm{e}-16$ & $0.76 \mathrm{e}-15$ \\
& 50 & $-0.4943 \mathrm{e}-13$ & $0.34 \mathrm{e}-14$ & 50 & $0.1844 \mathrm{e}-11$ & $0.30 \mathrm{e}-13$ \\
& 100 & $0.2466 \mathrm{e}-05$ & $0.34 \mathrm{e}-12$ & 100 & $-0.1503 \mathrm{e}-04$ & $0.32 \mathrm{e}-12$ \\
\hline$n=100$ & 1 & $-0.1820 \mathrm{e}-52$ & $0.30 \mathrm{e}-18$ & 1 & $-0.1412 \mathrm{e}-46$ & $0.16 \mathrm{e}-17$ \\
& 5 & $-0.3664 \mathrm{e}-48$ & $0.14 \mathrm{e}-16$ & 5 & $0.2170 \mathrm{e}-43$ & $0.19 \mathrm{e}-17$ \\
& 10 & $-0.2390 \mathrm{e}-45$ & $0.24 \mathrm{e}-16$ & 10 & $0.2489 \mathrm{e}-41$ & $0.11 \mathrm{e}-15$ \\
& 25 & $-0.4316 \mathrm{e}-40$ & $0.98 \mathrm{e}-16$ & 25 & $0.7255 \mathrm{e}-37$ & $0.28 \mathrm{e}-13$ \\
& 50 & $0.8123 \mathrm{e}-34$ & $0.50 \mathrm{e}-11$ & 50 & $-0.2162 \mathrm{e}-31$ & $0.18 \mathrm{e}-10$ \\
100 & $-0.2896 \mathrm{e}-26$ & $0.48 \mathrm{e}-06$ & 100 & $0.5772 \mathrm{e}-23$ & $0.18 \mathrm{e}-08$ \\
\hline
\end{tabular}

Then from (1.18) we can easily obtain the following recursion:

$$
p_{n, m}=\frac{p_{n-1, m-1}+4(m+1)(c-m-2) p_{n-1, m+1}}{2(2 m+n)}, \quad 0 \leq m \leq n,
$$

assuming $p_{n-1, m}=0$ for $m=-1, n, n+1$. After evaluating $P_{n-1}(c ; z)$ and computing $p_{n, m}$ by using (2.4), the array of coefficients $\left\{p_{n-1, m}\right\}, 0 \leq$ $m \leq n-1$, can be overwritten, so there is no need of storing many earlier coefficients for the next recursion step.

Another important aspect of the recurrence relations is the numerical stability. Considering the Perron-Kreuser theorem [7], it is easy to see that all the three linearly independent solutions of (1.8) behave as $\mathcal{O}\left(n^{\alpha}\right)$ for some $\alpha$. From this we conclude that the recursion for $A_{n}(a, c)$ is not unstable; only the usual rounding errors occur.

For the polynomials $P_{n}(c ; z)$ we notice a more difficult problem to prove the condition. The recurrence relation in (2.4) is rather simple, and intuitively we expect instability, because the $P_{n}(c ; z)$ become so small for large values of $n$.

To verify what happens we have collected some results in Table 1 from numerical experiments. We have computed in Maple the polynomials $P_{n}(c ; z)$ 
for $c= \pm \frac{17}{4}$ and several exact values of $z$ by using the generating function in (1.19). Next to this we have used a finite precision algorithm based on the recursion in $(2.4)$ to compute $\widetilde{P}_{n}(c ; z)$, using $c=4.25$ and values of $z$ in finite precision with Maple's parameter Digits $=20$. In the table we give the relative error $\delta=\left|P_{n}(c ; z) / \widetilde{P}_{n}(c ; z)-1\right|$ for $n=50$ and $n=100$. We conclude from the table that indeed instabilities occur in the recursion in (2.4) for larger $z$ and $n$.

\subsection{Asymptotic aspects of the expansions}

The expansions in (1.5) and (1.16) contain Bessel functions with argument $2 \sqrt{\kappa z}$, and in the literature asymptotic approximations of the Kummer functions are given in terms of $J$-Bessel functions, for $a \rightarrow-\infty$, see [13, p. 68]. For $a \rightarrow+\infty$ complete expansions in terms of the $I$-Bessel functions are given in $[13$, p. 80]. In these results the Bessel functions have the same argument as in (1.5) and (1.16), and also the orders of the first Bessel functions are the same. This indicates that the expansions in (1.5) and (1.16) may have an asymptotic character for large values of $a$.

In Tricomi's expansion (1.5) the coefficients are polynomials of $a$, and this makes the expansion not very useful as an asymptotic series for large $|a|$, unless $|z / \kappa|$ is quite small. In this connection, see also [13, p. 68], where it is shown that (1.5) has an asymptotic character for large values of $\kappa$ when $|z|=\mathcal{O}\left(|\kappa|^{\rho}\right)$ if $0 \leq \rho<\frac{1}{3}$.

Buchholz $[4, \S 7.4]$ derived the expansion in (1.16) to obtain asymptotic approximations of the Kummer function for large values of $\kappa=\frac{1}{2} c-a$, or for the Whittaker function $M_{\kappa, \mu}(z)$. Buchholz remarked that a Poincaré-type expansion for large $\kappa$ is difficult to obtain. Notice that in the expansion in (1.16) the parameter $\kappa$ only shows up in the argument of the Bessel function, and the parameter $c$ should be fixed. So, we expect that for the $F$-Kummer function the expansion in (1.16) can be used for large values of $|a|$.

Indeed, the asymptotic character of Buchholz' expansion (1.16) for large values of $\kappa$ follows from estimating the terms for fixed $n$. First we use [3, (9.1.60)] $\left|J_{\nu}(z)\right| \leq 1$ when $z \geq 0, \nu \geq 0$. This gives for the terms in (1.16) with $c-1+n \geq 0$ and $\kappa z>0$ the estimate (see also (1.1))

$$
\begin{aligned}
& 2^{-n} z^{n} P_{n}(c ; z) E_{c-1+n}(\kappa z)= \\
& 2^{-n} z^{n} P_{n}(c ; z)(\kappa z)^{-\frac{1}{2}(c-1+n)} J_{c-1+n}(2 \sqrt{\kappa z})=\mathcal{O}(z / \kappa)^{\frac{1}{2} n},
\end{aligned}
$$

as $\kappa \rightarrow+\infty$. It follows that (1.16) has an asymptotic character for large values of $\kappa$ (with $\kappa z>0$ ) when $|z|=o(\kappa)$, which, indeed, is much better 
Table 2: Relative errors $\delta$ in the computation of Buchholz' expansion (1.16) with terms up to and including $n=5$ and $n=10$ for $a=-500, c= \pm \frac{17}{4}$, and several values of $z$.

\begin{tabular}{rrcrc}
\hline & $c=17 / 4$ & \multicolumn{2}{c}{$c=-17 / 4$} \\
\hline & \multicolumn{1}{c}{$\delta$} & \multicolumn{1}{c}{$\delta$} & $\delta$ \\
\hline$n=5$ & 1 & $0.79 \mathrm{e}-12$ & 1 & $0.15 \mathrm{e}-10$ \\
& 5 & $0.12 \mathrm{e}-08$ & 5 & $0.35 \mathrm{e}-09$ \\
& 10 & $0.17 \mathrm{e}-07$ & 10 & $0.14 \mathrm{e}-07$ \\
& 25 & $0.50 \mathrm{e}-05$ & 25 & $0.13 \mathrm{e}-05$ \\
& 50 & $0.66 \mathrm{e}-04$ & 50 & $0.23 \mathrm{e}-03$ \\
\hline$n=10$ & 1 & $0.55 \mathrm{e}-19$ & 1 & $0.92 \mathrm{e}-18$ \\
& 5 & $0.16 \mathrm{e}-16$ & 5 & $0.71 \mathrm{e}-16$ \\
& 10 & $0.44 \mathrm{e}-17$ & 10 & $0.13 \mathrm{e}-14$ \\
& 25 & $0.15 \mathrm{e}-11$ & 25 & $0.19 \mathrm{e}-11$ \\
& 50 & $0.11 \mathrm{e}-06$ & 50 & $0.88 \mathrm{e}-09$ \\
\hline
\end{tabular}

than for Tricomi's expansion (1.5).

When $\kappa z<0$ the functions $E_{\nu}(z)$ become modified Bessel functions (see $(1.2))$. For these functions we have $[3,(9.6 .20)]$

$$
I_{\nu}(z)=\frac{1}{\pi} \int_{0}^{\pi} e^{z \cos \theta} \cos (\nu \theta) d \theta+\mathcal{O}(1)=e^{z}+\mathcal{O}(1)
$$

for $z \geq 0, \nu \geq 0$. So, also when $\kappa z<0$, we need the condition $|z|=o(\kappa)$.

In Table 2 we give the relative errors $\delta$ in the computation of Buchholz' expansion (1.16) with terms up to and including $n=5$ and $n=10$ for $a=-500, c= \pm \frac{17}{4}$, and several values of $z$. The relative error is computed by verifying the recurrence relation

$$
\begin{gathered}
c(c-1)_{1} F_{1}\left(\begin{array}{c}
a \\
c-1
\end{array} ; z\right)+c(1-c-z)_{1} F_{1}\left(\begin{array}{l}
a \\
c
\end{array} ; z\right)+ \\
z(c-a)_{1} F_{1}\left(\begin{array}{c}
a \\
c+1
\end{array} ; z\right)=0 .
\end{gathered}
$$

for the computed $F$-functions.

For similar negative values of $z$ and for $a=+500$ the results are the same, and we conclude that Buchholz' expansion (1.16) is very well suited for evaluating the Kummer function ${ }_{1} F_{1}(a ; c ; z)$ for large values of $|a|$. 


\section{Asymptotic expansion for $A_{n}(a, c)$}

We follow the same approach as in [10]. From (1.9) we obtain the Cauchy integral

$$
A_{n}(a, c)=\frac{1}{2 \pi i} \int_{\mathcal{C}} e^{(p-q) w}(1-w)^{p}(1+w)^{q} \frac{d w}{w^{n+1}}
$$

where

$$
p=-a, \quad q=a-c,
$$

and $\mathcal{C}$ is a circle around the origin with radius less than unity.

We observe that the singularities at \pm 1 are the sources for the main asymptotic contributions. We integrate around a circle with radius $R>1$, avoiding branch cuts running from \pm 1 to $\pm \infty$. The contribution from the circular arc is $\mathcal{O}\left(R^{-n}\right)$, which is exponentially small with respect to the main contributions. The loops around the branch cuts are denoted by $\mathcal{L}_{ \pm}$and the contributions from the loops by $I_{ \pm}$.

For the loop around the singular point $w=1$ we substitute $w=e^{s}$, and obtain

$$
I_{+}=\frac{2^{q} e^{p-q}}{2 \pi i} \int_{\mathcal{C}_{+}} g(s)\left(s e^{\pi i}\right)^{p} e^{-n s} d s
$$

where

$$
g(s)=\left(\frac{e^{s}-1}{s}\right)^{p}\left(\frac{e^{s}+1}{2}\right)^{q} e^{(p-q)\left(e^{s}-1\right)},
$$

and $\mathcal{C}_{+}$is the image of $\mathcal{L}_{+} \cdot \mathcal{C}_{+}$is a contour that encircles the origin in the clockwise fashion. For the asymptotic analysis we extend $\mathcal{C}_{+}$to $+\infty$. That is, we start the integration along the contour $\mathcal{C}_{+}$at $s=+\infty$, with ph $s=2 \pi$, turn around the origin in the clock-wise direction, and return to $+\infty$ with $\operatorname{ph} s=0 .^{2}$

We apply Barnes' lemma (also called Watson's lemma for loop integrals), see $[12$, p. 120$]$ and $[16$, p. 48$]$. We expand

$$
g(s)=\sum_{k=0}^{\infty} g_{k}(p, q) s^{k},
$$

substitute this in (3.3). This gives

$$
I_{+} \sim \frac{2^{q} e^{p-q}}{2 \pi i} \sum_{k=0}^{\infty} g_{k}(p, q) F_{k},
$$

\footnotetext{
${ }^{2}$ With ph $z$ we denote the phase (or argument) of the complex number $z$.
} 
where

$$
F_{k}=\frac{1}{2 \pi i} \int_{\mathcal{C}_{+}}\left(s e^{\pi i}\right)^{p} s^{k} e^{-n s} d s .
$$

To evaluate the integrals we turn the path by writing $s=e^{-\pi i} t$, and use the representation of the reciprocal gamma function in terms of the Hankel contour; see [14, p. 48]. The result is

$$
F_{k}=n^{-p-k-1} \frac{(-1)^{k}}{\Gamma(-p-k)}=n^{-p-k-1} \frac{(1+p)_{k}}{\Gamma(-p)} .
$$

This gives the expansion

$$
I_{+} \sim \frac{2^{q} e^{p-q}}{n^{p+1} \Gamma(-p)} \sum_{k=0}^{\infty} \frac{(1+p)_{k}}{n^{k}} g_{k}(p, q), \quad n \rightarrow \infty .
$$

The expansion for $I_{-}$, the contribution from the loop around the branch point $w=-1$, follows in a similar manner. It follows also by using the substitution $w \rightarrow-w$ in (3.1) and observing the symmetry with the previous case: change the sign of $\kappa$ (which involves interchanging $p$ and $q$ ), and include a factor $(-1)^{n}$. This gives

$$
I_{-} \sim(-1)^{n} \frac{2^{p} e^{q-p}}{n^{q+1} \Gamma(-q)} \sum_{k=0}^{\infty} \frac{(1+q)_{k}}{n^{k}} g_{k}(q, p), \quad n \rightarrow \infty .
$$

These two expansions give the compound expansion for $A_{n}$ :

$$
A_{n}(a, c) \sim I_{+}+I_{-}, \quad n \rightarrow \infty,
$$

and this holds for fixed values of $p$ and $q$ (or $\kappa$ and $c$ ).

The first few coefficients $g_{k}(p, q)$ are

$$
\begin{aligned}
g_{0}(p, q)= & 1, \quad g_{1}(p, q)=\frac{1}{2}(3 p-q), \\
g_{2}(p, q)= & \frac{1}{24}\left(13 p-9 q+3(3 p-q)^{2}\right), \\
g_{3}(p, q)= & \frac{1}{48}\left(8(p-q)+(3 p-q)(13 p-9 q)+(3 p-q)^{3}\right), \\
g_{4}(p, q)= & \frac{1}{5760}\left(30(13 p-9 q)(3 p-q)^{2}+15(3 p-q)^{4}+\right. \\
& \left.238 p-270 q-3090 p q+2285 p^{2}+885 q^{2}\right) .
\end{aligned}
$$

In Table 3 we give the relative errors $\delta$ when we use the expansions in (3.9) and (3.10) with terms up and including $k=3$, for several values of $a$, $c$ and $n$. We also give the values of $A_{n}(a, c)$. 
Table 3: Relative errors $\delta$ in the asymptotic expansions in(3.9) and (3.10) for several values of $a, c$ and $n$.

\begin{tabular}{ccccccc}
\hline & \multicolumn{3}{c}{$c=2.25$} & \multicolumn{3}{c}{$c=-2.25$} \\
\hline$n=50$ & $a$ & $A_{n}(a, c)$ & $\delta$ & $a$ & $A_{n}(a, c)$ & $\delta$ \\
\hline \multirow{6}{*}{$n=100$} & $0.4375 \mathrm{e}+1$ & $0.99 \mathrm{e}-8$ & 0.5 & $0.2036 \mathrm{e}-1$ & $0.22 \mathrm{e}-5$ \\
& 2.5 & $0.2260 \mathrm{e}+2$ & $0.97 \mathrm{e}-7$ & 2.5 & $0.6040 \mathrm{e}+1$ & $0.87 \mathrm{e}-6$ \\
& 5.0 & $0.1432 \mathrm{e}+4$ & $0.26 \mathrm{e}-3$ & 5.0 & $0.4164 \mathrm{e}+3$ & $0.59 \mathrm{e}-3$ \\
& 7.5 & $0.3041 \mathrm{e}+5$ & $0.22 \mathrm{e}-1$ & 7.5 & $0.9474 \mathrm{e}+4$ & $0.34 \mathrm{e}-1$ \\
\hline & 0.5 & $0.7153 \mathrm{e}+1$ & $0.51 \mathrm{e}-9$ & 0.5 & $0.1456 \mathrm{e}-1$ & $0.28 \mathrm{e}-7$ \\
& 2.5 & $0.6054 \mathrm{e}+2$ & $0.66 \mathrm{e}-8$ & 2.5 & $0.1571 \mathrm{e}+2$ & $0.61 \mathrm{e}-7$ \\
& 5.0 & $0.1690 \mathrm{e}+5$ & $0.22 \mathrm{e}-4$ & 5.0 & $0.4604 \mathrm{e}+4$ & $0.54 \mathrm{e}-4$ \\
& 7.5 & $0.1351 \mathrm{e}+7$ & $0.26 \mathrm{e}-2$ & 7.5 & $0.3842 \mathrm{e}+6$ & $0.44 \mathrm{e}-2$ \\
\hline
\end{tabular}

\subsection{Integer values of $p$ or $q$}

The expansion for $I_{+}$vanishes when $p=0,1,2, \ldots$, and similar for $I_{-}$when $q=0,1,2, \ldots$ This is in agreement with the different nature of the points $w= \pm 1$ in (3.1). When both $p$ and $q$ are equal to non-negative integers, the singularities disappear, and $A_{n}$ becomes exponentially small for large values of $n$. In other words, the expansion in (1.9) converges for all finite complex $w$ when $\kappa-\frac{1}{2} c=j$ and $-\kappa-\frac{1}{2} c=k, j, k=0,1,2, \ldots$.

For the $a$ and $c$ parameters of the ${ }_{1} F_{1}$ this means (see (1.6)) $a=-j$, $c-a=-k$. When $a=-j$ the ${ }_{1} F_{1}$ reduces to a polynomial; when $c-a=-k$ it reduces to an exponential function times a polynomial, as follows from

$$
{ }_{1} F_{1}\left(\begin{array}{l}
a \\
c
\end{array} ; z\right)=e^{z}{ }_{1} F_{1}\left(\begin{array}{c}
c-a \\
c
\end{array} ;-z\right) .
$$

For an interpretation of ${ }_{1} F_{1}(a ; c ; z) / \Gamma(c)$ for $c=0,-1,-2, \ldots$, see $(1.21)$.

When $p$ and $q$ are both equal to non-negative integers we proceed as follows (for $p=q$ we refer to (1.10)). We expand

$$
(1-w)^{p}(1+w)^{q}=\left(1-w_{0}\right)^{p}\left(1+w_{0}\right)^{q} \sum_{k=0}^{\max (p, q)} c_{k}\left(w-w_{0}\right)^{k},
$$

where $w_{0}=n /(p-q)$. This value $w_{0}$ is the saddle point of the function $e^{(p-q) w} w^{-n}$. 
Substituting the expansion (3.14) in (3.1) gives

$$
A_{n}(a, c)=\left(1-w_{0}\right)^{p}\left(1+w_{0}\right)^{q} \frac{(p-q)^{n}}{n !} \sum_{k=0}^{\max (p, q)} \frac{c_{k}}{(p-q)^{k}} \phi_{k},
$$

where

$$
\phi_{k}=\frac{(p-q)^{k-n} n !}{2 \pi i} \int_{\mathcal{C}}\left(w-w_{0}\right)^{k} e^{(p-q) w} \frac{d w}{w^{n+1}},
$$

These $\phi_{k}$ are polynomials in $n$ and can be obtained from the recursion relation

$$
\phi_{k+1}=-k\left(\phi_{k}+n \phi_{k-1}\right), \quad k \geq 0, \quad \phi_{0}=1 .
$$

The first few are

$$
\phi_{1}=0, \quad \phi_{2}=-n, \quad \phi_{3}=2 n, \quad \phi_{4}=3 n(n-2) .
$$

From (3.17) and induction it follows that the degrees of $\phi_{2 k}$ and $\phi_{2 k+1}$ equals $k$. This, together with the fact that $c_{k}=\mathcal{O}\left(n^{-k}\right)$ shows that the finite expansion in (3.15) has an asymptotic character for large values of $n$.

\subsection{An alternative expansion for $A_{n}(a, c)$}

In the method for obtaining the expansions for $I_{ \pm}$by using (3.1) the main contributions come from the points $w= \pm 1$. In this section we expand $e^{(p-q) w}$ in a two-point Taylor expansion at $w= \pm 1$. In this way an alternative asymptotic representation can be obtained. For more details on two-point Taylor expansions we refer to $[8,9]$.

We write

$$
f(w)=e^{r w}=\sum_{m=0}^{\infty}\left(a_{m}+w b_{m}\right)\left(w^{2}-1\right)^{m}, \quad r=p-q .
$$

The function $f$ satisfies $f^{\prime}=r f$, hence we have the relations

$$
\begin{aligned}
& 2(m+1) a_{m+1}=r b_{m}, \\
& 2(m+1) b_{m+1}=r a_{m}-(2 m+1) b_{m},
\end{aligned}
$$

$m=0,1,2, \ldots$, with starting values $a_{0}=\cosh r, b_{0}=\sinh r$.

Putting the expansion in (3.1) we obtain

$$
A_{n}(a, c)=\sum_{m=0}^{\infty}(-1)^{m}\left(a_{m} H_{m}(p, q ; n)+b_{m} H_{m}(p, q ; n-1)\right),
$$


where

$$
H_{m}(p, q ; n)=\frac{1}{2 \pi i} \int_{\mathcal{C}}(1-w)^{p+m}(1+w)^{q+m} \frac{d w}{w^{n+1}} .
$$

This function can be written in the form

$$
H_{m}(p, q ; n)=\sum_{k=0}^{n}(-1)^{k}\left(\begin{array}{c}
p+m \\
k
\end{array}\right)\left(\begin{array}{c}
q+m \\
n-k
\end{array}\right) .
$$

By symmetry we have

$$
H_{m}(p, q ; n)=(-1)^{n} H_{m}(q, p ; n) .
$$

For large $n$ the representation in (3.23) is not a convenient one, and we write it as a Gauss hypergeometric function. We use the loop integral given in $[14$, p. 111].

First we write

$$
H_{m}(p, q ; n)=\frac{2^{p+m}}{2 \pi i} \int_{0}^{(1+)}\left(1-\frac{1}{2} w\right)^{p+m} w^{q+m} \frac{d w}{(w-1)^{n+1}},
$$

and obtain

$$
H_{m}(p, q ; n)=\frac{2^{p+m} \Gamma(q+m+1)}{\Gamma(q+m+1-n) n !}{ }_{2} F_{1}\left(\begin{array}{c}
-p-m, q+m+1 \\
q+m+1-n
\end{array} ; \frac{1}{2}\right) .
$$

By using the many transformations for the Gauss functions we can write these in different forms, for example, by using

$$
{ }_{2} F_{1}\left(\begin{array}{c}
a, b \\
c
\end{array} ; z\right)=(1-z)^{-a} F_{1}\left(\begin{array}{c}
a, c-b \\
c
\end{array} \frac{z}{z-1}\right) .
$$

This gives

$$
H_{m}(p, q ; n)=\frac{\Gamma(q+m+1)}{\Gamma(q+m+1-n) n !}{ }_{2} F_{1}\left(\begin{array}{l}
-p-m,-n \\
q+m+1-n
\end{array} ;-1\right) .
$$

Because $n$ is an integer, this ${ }_{2} F_{1}$ is a finite sum, and it can be written in the form (3.23). Another form is

$$
\begin{aligned}
& H_{m}(p, q ; n)=\Gamma(n-p-q-2 m) \times \\
& \left(\frac{(-1)^{n} 2^{p+m}}{\Gamma(-q-m) \Gamma(n-p-m+1)}{ }_{2} F_{1}\left(\begin{array}{c}
-p-m, q+m+1 \\
n-p-m+1
\end{array} ; \frac{1}{2}\right)+\right. \\
& \left.\frac{2^{q+m}}{\Gamma(-p-m) \Gamma(n-q-m+1)}{ }_{2} F_{1}\left(\begin{array}{c}
-q-m, p+m+1 \\
n-q-m+1
\end{array} ; \frac{1}{2}\right)\right) \text {. }
\end{aligned}
$$


The symmetry relation (3.24) gives other forms of (3.26) and (3.28), whereas (3.29) remains the same.

The Gauss function in (3.28) reduces to a finite sum when $p+m=$ $0,-1,-2, \ldots$ (and the same for the version for $H_{m}(q, p ; n)$ when $q+m$ is a non-positive integer).

The representation in (3.29) is very convenient for evaluating $H_{m}(p, q ; n)$ for large values of $n$, because the series of the Gauss functions are convergent asymptotic series for large $n$. Both Gauss functions behave like $1+\mathcal{O}(1 / n)$, and hence, by using $\Gamma(n+a) / \Gamma(n+b)=n^{a-b}(1+\mathcal{O}(1 / n))$,

$$
H_{m}(p, q ; n)=2^{m} n^{-m-1}\left(\frac{(-1)^{n} 2^{p} n^{-q}}{\Gamma(-q-m)}+\frac{2^{q} n^{-p}}{\Gamma(-p-m)}+\mathcal{O}\left(n^{-1}\right)\right),
$$

as $n \rightarrow \infty$, with $p, q, m$ fixed, and $p+m$ and $q+m$ not both equal to a non-positive integer.

This shows the asymptotic nature of the expansion in (3.21), and because the expansion in (3.19) converges absolute and uniformly for $w$ in compact sets of $\mathbb{C}$, the expansion in (3.21) is convergent as well. The coefficients $a_{m}$ and $b_{m}$ can be computed by using the recursion in (3.20). For $H_{m}$ a threeterm recurrence relation also exists (because it is related with the Gauss function. To find this recursion, we start with the relation

$$
A_{2} F_{1}\left(\begin{array}{c}
a-1, b \\
c+1
\end{array} ; z\right)+B_{2} F_{1}\left(\begin{array}{c}
a, b \\
c
\end{array} ; z\right)+C_{2} F_{1}\left(\begin{array}{c}
a-2, b \\
c+2
\end{array} ; z\right)=0
$$

By using several contiguous relations $[3$, p. 558] we find

$$
A=A_{0}+A_{1} z+A_{2} z^{2}+A_{3} z^{3}
$$

where

$$
\begin{aligned}
& A_{0}=-(a-1)(a-2) c(c+1), \\
& A_{1}=-(a-1)(a-2)(c+1)(a-4 c-3+3 b), \\
& A_{2}=-(c+1)(b-c)(b-c-1)(4 a-c-6), \\
& A_{3}=(c+1)(b-c)(b-c-1)(a-b-1),
\end{aligned}
$$

and

$$
\begin{aligned}
& B=(a-1) c(c+1)(a-2+(b-1-c) z)(1-z)^{2}, \\
& C=(c+1-b)(a-c-3)(a-c-2)(a-1+z(b-c)) z .
\end{aligned}
$$


Using representation (3.28) we find

$$
P H_{m+2}(p, q ; n)=Q H_{m+1}(p, q ; n)+R H_{m}(p, q ; n),
$$

where

$$
\begin{aligned}
P= & (p+q+2 m-n+4)(p+q+2 m-n+3), \\
Q= & p^{2}+q^{2}+6 p q+11(p+q+2 m)+8 m(p+q+m)+ \\
& 16-2 n(p+q)-4 n m-6 n, \\
R= & -4(p+m+1)(q+m+1) .
\end{aligned}
$$

\section{Asymptotic expansion for $\boldsymbol{P}_{n}(c ; z)$}

From (1.19) we obtain

$$
(-1)^{n} P_{n}(c ; z)=\frac{1}{2 \pi i} \int_{\mathcal{C}} e^{-\frac{1}{2} z(\operatorname{coth} w-1 / w)}\left(\frac{\sinh w}{w}\right)^{c-2} \frac{d w}{w^{n+1}},
$$

where $\mathcal{C}$ is a circle around the origin with radius smaller than $\pi$. The singularities of the integrand of (4.1) are of a different type, compared with those of $\S 3$ : at $w= \pm \pi i$ we have essential singularities, and we need Bessel functions for the asymptotic form.

We observe that for $z=0$ we have

$$
P_{2 n+1}(c ; 0)=0, \quad P_{2 n}(c ; 0)=2^{2 n} \frac{B_{2 n}^{-\mu}\left(-\frac{1}{2} \mu\right)}{(2 n) !}, \quad \mu=c-2 .
$$

where $B_{n}^{\mu}(z)$ is the generalized Bernoulli polynomial defined by the generating series

$$
\frac{w^{\mu} e^{w z}}{\left(e^{w}-1\right)^{\mu}}=\sum_{n=0}^{\infty} \frac{B_{n}^{\mu}(z)}{n !} w^{n}, \quad|w|<2 \pi .
$$

The large $n$ asymptotic of the generalized Bernoulli polynomial can be found in $[10, \S 2.3]$.

When $z \neq 0$ we first transform $w \rightarrow i w$, because we like to have the singularities on the real line, as for $A_{n}(a, c)$ in $\S 3$. We obtain

$$
P_{n}(c ; z)=i^{n} P_{n}^{\mu}(i z),
$$

where

$$
P_{n}^{\mu}(z)=\frac{1}{2 \pi i} \int_{\mathcal{C}} e^{z(\cot w-1 / w) / 2}\left(\frac{\sin w}{w}\right)^{\mu} \frac{d w}{w^{n+1}}, \quad \mu=c-2 .
$$


We enlarge the radius of the circle in (4.5) and integrate around a circle with radius $R, \pi<R<2 \pi$, avoiding branch cuts running from $\pm \pi$ to $\pm \infty$. The contribution from the circular arc is $\mathcal{O}\left(R^{-n}\right)$, which is exponentially small with respect to the main contributions. The loops around the branch cuts from $\pm \pi$ to $\pm \infty$ are denoted by $\mathcal{L}_{ \pm}$and the contributions from the loops by $I_{ \pm}$.

\subsection{The evaluation of $I_{+}$}

For the singularity at $+\pi$ we substitute $w=\pi e^{s}$. We have, as $s \rightarrow 0$,

$$
\begin{aligned}
& \sin w=\sin \pi\left(1-e^{s}\right)=-\pi s-\frac{1}{2} \pi s^{2}+\mathcal{O}\left(s^{3}\right), \\
& \cot w=\cot \pi\left(e^{s}-1\right)=\frac{1}{\pi s}-\frac{1}{2 \pi}+\mathcal{O}(s) .
\end{aligned}
$$

After these preparations we can write

$$
I_{+}=\frac{e^{-3 z /(4 \pi)} \pi^{-n}}{2 \pi i} \int_{\mathcal{L}_{+}} e^{\phi(s)} f(s)(-s)^{\mu} e^{z /(2 \pi s)-n s} d s
$$

where

$$
\begin{aligned}
& \phi(s)=\frac{1}{2} z\left(\cot \pi\left(e^{s}-1\right)-\frac{1}{\pi s}+\frac{1}{2 \pi}-\frac{1}{\pi}\left(e^{-s}-1\right)\right), \\
& f(s)=\left(\frac{\sin \pi\left(1-e^{s}\right)}{-s \pi e^{s}}\right)^{\mu} .
\end{aligned}
$$

By writing $s=e^{\pi i} t$ we turn the contour to the negative real axis and obtain

$$
I_{+}=\frac{e^{-3 z /(4 \pi)} \pi^{-n}}{2 \pi i} \int_{\mathcal{L}} g(t) t^{\mu} e^{-z /(2 \pi t)+n t} d t
$$

where $\mathcal{L}$ is the Hankel contour and $g(t)=e^{\phi(-t)} f(-t)$. That is,

$$
\begin{gathered}
g(t)=\exp \left(-\frac{1}{2} z\left(\cot \pi\left(1-e^{-t}\right)-\frac{1}{\pi t}-\frac{1}{2 \pi}+\frac{1}{\pi}\left(e^{t}-1\right)\right)\right) \times \\
\left(\frac{e^{t} \sin \pi\left(1-e^{-t}\right)}{t \pi}\right)^{\mu} .
\end{gathered}
$$

The function $g(t)$ in (4.10) is analytic at the origin and we can expand it in powers of $t$, interchange integration and summation, and obtain a series of 
Bessel functions with decreasing orders. Instead we use integration by parts, and obtain a compound expansion in which two Bessel functions occur.

To see the connection with Bessel functions we mention the integral representation (see [14, p. 277])

$$
\left(\frac{1}{2} z\right)^{-\nu} J_{\nu}(z)=\frac{1}{2 \pi i} \int_{\mathcal{L}} e^{s-z^{2} /(4 s)} \frac{d s}{s^{\nu+1}},
$$

valid for all complex $z$ and $\nu$. In our approach we need the integral

$$
\Phi_{\mu}(z)=\frac{1}{2 \pi i} \int_{\mathcal{L}} e^{n t-z /(2 \pi t)} t^{\mu} d t=\left(\frac{z}{2 \pi n}\right)^{(\mu+1) / 2} J_{-\mu-1}(\sqrt{2 n z / \pi}),
$$

which can be written in the form (see (1.1))

$$
\Phi_{\mu}(z)=n^{-\mu-1} E_{-\mu-1}\left(\frac{n z}{2 \pi}\right),
$$

showing that $\Phi_{\mu}(z)$ is an entire function of its argument $z$.

Next we write

$$
g(t)=g(0)+g^{\prime}(0) t+t^{2} h(t)
$$

where the derivative is with respect to $t$. Substituting this in (4.9) we obtain, after integrating by parts,

$$
\begin{aligned}
& I_{+}=\frac{e^{-3 z /(4 \pi)}}{\pi^{n}} \times \\
& \left(g(0) \Phi_{\mu}(z)+g^{\prime}(0) \Phi_{\mu+1}(z)+\frac{1}{2 \pi i n} \int_{\mathcal{L}} g_{1}(t) t^{\mu} e^{-z /(2 \pi t)+n t} d t\right),
\end{aligned}
$$

where

$$
g_{1}(t)=-\left(t^{2} h^{\prime}(t)+\frac{z}{2 \pi} h(t)+(\mu+2) t h(t)\right) .
$$

Continuing this procedure, we obtain

$$
I_{+} \sim \frac{e^{-3 z /(4 \pi)}}{\pi^{n}}\left(\Phi_{\mu}(z) \sum_{k=0}^{\infty} \frac{A_{k}(\mu, z)}{n^{k}}+\Phi_{\mu+1}(z) \sum_{k=0}^{\infty} \frac{B_{k}(\mu, z)}{n^{k}}\right),
$$

where $A_{k}(\mu, z)=g_{k}(0)$ and $B_{k}(\mu, z)=g_{k}^{\prime}(0)$ and the functions $g_{k}(t)$ are defined by

$$
g_{k+1}(t)=-\left(t^{2} h_{k}^{\prime}(t)+\frac{z}{2 \pi} h_{k}(t)+(\mu+2) t h_{k}(t)\right), \quad k=0,1,2, \ldots,
$$

with $g_{0}(t)=g(t)$. The functions $h_{k}(t)$ follow from

$$
g_{k}(t)=g_{k}(0)+g_{k}^{\prime}(0) t+t^{2} h_{k}(t), \quad k=0,1,2, \ldots
$$




\subsection{The evaluation of $I_{-}$}

For the singularity at $-\pi$ we can use a symmetry argument by observing that we can transform in (4.5) $w \rightarrow-w$, which is equivalent with changing the sign of $z$ and adding a front factor $(-1)^{n}$. This gives

$$
I_{-} \sim(-1)^{n} \frac{e^{3 z /(4 \pi)}}{\pi^{n}}\left(\Phi_{\mu}(-z) \sum_{k=0}^{\infty} \frac{A_{k}(\mu,-z)}{n^{k}}+\Phi_{\mu+1}(-z) \sum_{k=0}^{\infty} \frac{B_{k}(\mu,-z)}{n^{k}}\right) .
$$

\subsection{The coefficients of the expansions}

The first few coefficients in the expansion (4.17) are given by

$$
\begin{gathered}
A_{0}(\mu, z)=1, \quad B_{0}(\mu, z)=\frac{1}{24 \pi}\left(12 \mu \pi-13 z+4 z \pi^{2}\right) \\
A_{1}(\mu, z)=-\frac{z}{2304 \pi^{3}}\left(-96 z \pi^{3}-288 z \pi-104 z^{2} \pi^{2}-312 z \mu \pi\right. \\
\left.+169 z^{2}+16 z^{2} \pi^{4}+96 z \pi^{3} \mu+144 \mu^{2} \pi^{2}-192 \mu \pi^{4}+48 \mu \pi^{2}\right) \\
B_{1}(\mu, z)=-\frac{1}{829440 \pi^{4}}\left(8640 z \mu^{3} \pi^{3}-51840 z^{2} \pi^{4} \mu-28080 z^{2} \mu^{2} \pi^{2}\right. \\
+2880 z^{3} \pi^{5} \mu+27648 z^{2} \pi^{6}-10985 z^{4}-138240 z \pi^{5}-5760 z^{3} \pi^{5} \\
+10140 z^{4} \pi^{2}-3120 z^{4} \pi^{4}+320 z^{4} \pi^{6}+1440 z^{3} \pi^{3}+56160 z^{3} \pi \\
+34560 z \pi^{5} \mu^{2}-216000 z \pi^{3} \mu^{2}+60480 z^{2} \pi^{2} \mu+30420 z^{3} \mu \pi \\
-18720 z^{3} \pi^{3} \mu+138240 z \pi^{5} \mu+8640 z^{2} \pi^{4} \mu^{2}+69120 \mu \pi^{4} \\
-656640 z \pi^{3} \mu-276480 \mu \pi^{6}+103680 \mu^{3} \pi^{4}+241920 \mu^{2} \pi^{4} \\
\left.-138240 \mu^{2} \pi^{6}-414720 z \pi^{3}+209088 z^{2} \pi^{2}-138240 z^{2} \pi^{4}\right) .
\end{gathered}
$$

\subsection{The complete expansion for $P_{n}(c ; z)$}

Using (4.17) and (4.20), we obtain

$$
\begin{aligned}
& P_{n}^{\mu}(z) \sim \frac{e^{-3 z /(4 \pi)}}{\pi^{n}}\left(\Phi_{\mu}(z) \sum_{k=0}^{\infty} \frac{A_{k}(\mu, z)}{n^{k}}+\Phi_{\mu+1}(z) \sum_{k=0}^{\infty} \frac{B_{k}(\mu, z)}{n^{k}}\right)+ \\
& (-1)^{n} \frac{e^{3 z /(4 \pi)}}{\pi^{n}}\left(\Phi_{\mu}(-z) \sum_{k=0}^{\infty} \frac{A_{k}(\mu,-z)}{n^{k}}+\Phi_{\mu+1}(-z) \sum_{k=0}^{\infty} \frac{B_{k}(\mu,-z)}{n^{k}}\right),
\end{aligned}
$$


Table 4: Relative errors $\delta$ in the asymptotic expansions in (4.24) for several values of $z, \mu$ and $n$.

\begin{tabular}{rrccrcc}
\hline \multicolumn{3}{c}{$\mu=2.25$} & \multicolumn{3}{c}{$\mu=-2.25$} \\
\hline$n=50$ & \multicolumn{1}{c}{$P_{n}^{\mu}(z)$} & $\delta$ & $z$ & $P_{n}^{\mu}(z)$ & $\delta$ \\
\hline & 0.5 & $0.1057 \mathrm{e}-28$ & $0.56 \mathrm{e}-4$ & 0.5 & $0.7438 \mathrm{e}-22$ & $0.28 \mathrm{e}-3$ \\
& 2.5 & $0.5370 \mathrm{e}-25$ & $0.62 \mathrm{e}-3$ & 2.5 & $0.4067 \mathrm{e}-20$ & $0.38 \mathrm{e}-2$ \\
& 5.0 & $0.1129 \mathrm{e}-22$ & $0.26 \mathrm{e}-2$ & 5.0 & $0.1431 \mathrm{e}-18$ & $0.22 \mathrm{e}-1$ \\
& 10.0 & $0.1544 \mathrm{e}-19$ & $0.75 \mathrm{e}-2$ & 10.0 & $0.3374 \mathrm{e}-16$ & $0.25 \mathrm{e}-0$ \\
\hline$n=100$ & 0.5 & $0.3618 \mathrm{e}-53$ & $0.41 \mathrm{e}-5$ & 0.5 & $0.7474 \mathrm{e}-46$ & $0.70 \mathrm{e}-4$ \\
& 2.5 & $0.9816 \mathrm{e}-49$ & $0.20 \mathrm{e}-3$ & 2.5 & $0.3156 \mathrm{e}-43$ & $0.95 \mathrm{e}-3$ \\
& 5.0 & $0.9190 \mathrm{e}-46$ & $0.78 \mathrm{e}-3$ & 5.0 & $0.5282 \mathrm{e}-41$ & $0.55 \mathrm{e}-2$ \\
& 10.0 & $0.1129 \mathrm{e}-41$ & $0.21 \mathrm{e}-2$ & 10.0 & $0.1181 \mathrm{e}-37$ & $0.58 \mathrm{e}-1$ \\
\hline
\end{tabular}

where $\Phi_{\mu}(z)$ is defined in (4.12) and (4.13). This expansion holds for $n \rightarrow \infty$, uniformly with respect to bounded complex $z$ and $\mu$. In particular the expansion is valid for small $z$. When $z$ is bounded away from 0 we can expand the Bessel functions (see (4.12)) in terms of their large argument asymptotic expansions.

In Table 4 we give the relative errors $\delta$ when we use the expansions in (4.24) with terms up and including $k=2$, for several values of $z, \mu$ and $n$. We also give the values of $P_{n}^{\mu}(z)$.

For the expansion in (1.16) we need the relation in (4.4), and we have

$$
{ }_{1} F_{1}\left(\begin{array}{l}
a \\
c
\end{array} ; z\right)=\Gamma(c) e^{\frac{1}{2} z} \sum_{n=0}^{\infty} 2^{-n}(i z)^{n} P_{n}^{\mu}(i z) E_{c-1+n}(\kappa z) .
$$

When we use this representation with $z$ real and want to estimate the rate of convergence, we have to write in $(4.24) \Phi_{\mu}(i z)$ and the coefficients in the expansions in real and imaginary parts. For example, when we write

$$
E_{\nu}(i y)=E_{\nu}^{(r)}(y)+i E_{\nu}^{(i)}(y)
$$

and use the first term approximation

$$
P_{n}(c ; z) \sim \frac{i^{n}}{\pi^{n}}\left(e^{-3 i z /(4 \pi)} \Phi_{\mu}(i z)+(-1)^{n} e^{3 i z /(4 \pi)} \Phi_{\mu}(-i z)\right),
$$


then we have, using (4.13),

$$
\begin{aligned}
& P_{2 n}(c ; z) \sim \frac{2(-1)^{n}(2 n)^{1-c}}{\pi^{2 n}}\left(\cos \zeta E_{1-c}^{(r)}(y)+\sin \zeta E_{1-c}^{(i)}(y)\right), \\
& P_{2 n+1}(c ; z) \sim \frac{2(-1)^{n+1}(2 n+1)^{1-c}}{\pi^{2 n+1}}\left(\cos \zeta E_{1-c}^{(i)}(y)-\sin \zeta E_{1-c}^{(r)}(y)\right),
\end{aligned}
$$

where

$$
\zeta=\frac{3 z}{4 \pi}, \quad y=\frac{n z}{2 \pi} .
$$

\section{Concluding remarks}

Comparing Tricomi's expansion (1.5) with Buchholz' expansion (1.16) we prefer the latter because of the faster convergence properties. In addition, it performs better in the asymptotic evaluation of the Kummer function for large values of $a$. The computation of $A_{n}(c ; z)$ is straightforward, without instabilities, whereas $P_{n}(c ; z)$ are more difficult to evaluate, and the computation in finite arithmetic may become unstable.

Tricomi's expansion (1.5) has been used in [11] for the computation of parabolic cylinder functions, and we expect better representations by using Buchholz' expansion.

Acknowledgments. The authors thank the referees for their valuable comments. The Gobierno of Navarra, Res.07/05/2008, is acknowledged for its financial support. JLL acknowledges financial support from Ministerio de Educación y Ciencia, project MTM2007-63772. NMT acknowledges financial support from Ministerio de Ciencia e Innovación, projects MTM200609050 and MTM2009-11686.

\section{References}

[1] J. Abad and J. Sesma. Buchholz polynomials: a family of polynomials relating solutions of confluent hypergeometric and Bessel equations. $J$. Comput. Appl. Math., 101(1-2):237-241, 1999.

[2] Julio Abad and Javier Sesma. A new expansion of the confluent hypergeometric function in terms of modified Bessel functions. J. Comput. Appl. Math., 78(1):97-101, 1997.

[3] M. Abramowitz and I. A. Stegun. Handbook of Mathematical Functions with Formulas, Graphs, and Mathematical Tables, volume 55 of National 
Bureau of Standards Applied Mathematics Series. U.S. Government Printing Office, Washington, 1964.

[4] H. Buchholz. The Confluent Hypergeometric Function with Special Emphasis on Its Applications. Translated from the German by H. Lichtblau and K. Wetzel. Springer Tracts in Natural Philosophy, Vol. 15. SpringerVerlag New York Inc., New York, 1969.

[5] C. Chiccoli, S. Lorenzutta, and G. Maino. A note on a Tricomi expansion for the generalized exponential integral and related functions. Nuovo Cimento B (11), 103(5):563-568, 1989.

[6] Amparo Gil, Javier Segura, and Nico M. Temme. Numerical Methods for Special Functions. Society for Industrial and Applied Mathematics (SIAM), Philadelphia, PA, 2007.

[7] P. Kreuser. Über das Verhalten der Integrale homogener linearer Differenzengleichungen im Unendlichen. PhD thesis, Diss. Tübingen, $48 \mathrm{~S}$, 1914.

[8] José L. López and Nico M. Temme. Two-point Taylor expansions of analytic functions. Stud. Appl. Math., 109(4):297-311, 2002.

[9] José L. López and Nico M. Temme. Multi-point Taylor expansions of analytic functions. Trans. Amer. Math. Soc., 356(11):4323-4342 (electronic), 2004.

[10] José L. López and Nico M. Temme. Large degree asymptotics of generalized Bernoulli and Euler polynomials. J. Math. Anal. Appl., 363(1):197$208,2010$.

[11] G. Maino, E. Menapace, and A. Ventura. Computation of parabolic cylinder functions by means of a Tricomi expansion. J. Comput. Phys., 40(2):294-304, 1981.

[12] F. W. J. Olver. Asymptotics and Special Functions. AKP Classics. A K Peters Ltd., Wellesley, MA, 1997. Reprint, with corrections, of original Academic Press edition, 1974.

[13] L. J. Slater. Confluent hypergeometric functions. Cambridge University Press, New York, 1960.

[14] N. M. Temme. Special Functions. A Wiley-Interscience Publication. John Wiley \& Sons Inc., New York, 1996. An introduction to the classical functions of mathematical physics. 
[15] Francesco Tricomi. Sulle funzioni ipergeometriche confluenti. Ann. Mat. Pura Appl. (4), 26:141-175, 1947.

[16] R. Wong. Asymptotic Approximations of Integrals, volume 34 of Classics in Applied Mathematics. Society for Industrial and Applied Mathematics (SIAM), Philadelphia, PA, 2001. Corrected reprint of the 1989 original. 